ISSN 0103-8478

\title{
Influência do ambiente no desenvolvimento de colônias iniciais de formigas cortadeiras (Atta sexdens rubropilosa)
}

\author{
Influence of environment on the development of initial colonies of leaf \\ cutting ants (Atta sexdens rubropilosa)
}

\author{
Roberto da Silva Camargo ${ }^{I^{*}}$ Juliana Amorim Fonseca $^{\mathrm{I}}$ \\ Juliane Floriano Santos Lopes ${ }^{\mathrm{I}}$ Luiz Carlos Forti ${ }^{\mathrm{II}}$
}

\section{RESUMO}

Anualmente, as colônias maduras de Atta spp. produzem formigas aladas, machos e fêmeas, que abandonam a colônia na qual foram produzidas para formarem novas colônias $e$, dessa forma, continuarem a perpetuação da espécie. A fundação de colônias de Atta spp. sempre envolve grande esforço da rainha, única responsável pelo cultivo do fungo e pelos cuidados consigo mesma e com sua prole. Nesse período de fundação ocorre o consumo de grande parte de suas reservas. O presente estudo investigou como o ambiente influencia o desenvolvimento das colônias recém-fundadas de Atta sexdens rubropilosa. Para tanto, foram comparadas colônias de campo coletadas cinco meses após a revoada e colônias mantidas em laboratório desde o voo nupcial. Foram analisados o peso e o tamanho da população (número de operárias, de larvas, de pupas e de ovos), o tamanho das operárias, o volume e peso do jardim de fungo e o esforço para a fundação dos ninhos (teor de lipídeos e peso das rainhas). Comparadas às colônias de campo, as colônias de laboratório apresentaram maiores valores no tamanho e no peso da população, no volume $e$ no peso do jardim de fungo. As medidas das operárias, por classe de tamanho, não variaram entre as colônias de campo e de laboratório. $O$ esforço da fundação dos ninhos foi maior nas colonias de campo, cujas rainhas apresentaram menor porcentagem de lipídeos e menor peso em relação às colônias de laboratório. Esses resultados demonstram que o ambiente exerce forte influência no desenvolvimento das colônias durante a fase de fundação, bem como sobre o status fisiológico das rainhas, sendo, portanto, a sobrevivência, o desenvolvimento e o sucesso da colônia reflexos das condições do ambiente e do esforço da rainha.

Palavras chave: escavação do ninho, fundação da colônia e formigas.

\section{ABSTRACT}

Annually, mature colonies of Atta ants produce winged males and females, where leave the colony in which they were produced to form new colonies and thus continue the species perpetuation. The foundation of Atta spp. colonies always involves high queen effort, solely responsible for growing the fungus and the care with itself and its offspring. During the foundation there is consumption of body reserves. This study investigated how the environment influences the development of newly founded colonies of leaf cutting ant, Atta sexdens rubropilosa. Therefore, it was compared field colonies collected five months after the nuptial flight and colonies maintained in the laboratory since the nuptial flight. It was analyzed the weight and size of the population (number of workers, larvae, pupae and eggs), the workers size, the volume and weight of the fungus garden and queen effort for the nest foundation (lipid content and weight of queens). In comparison to the field colonies, the laboratory colonies showed higher values in the weight and size of population, the volume and weight of the fungus garden. Measures of workers by class size did not vary between colonies from field and laboratory. The queen effort for nest foundation was higher in field colonies, which showed a lower percentage of fat and lower weight compared to laboratory colonies. These results show that the environment has a strong influence on the colonies development during the foundation, as well as on the physiological status of the queens, and therefore the survival, development and success of the colony, it is a reflection of the queen efforts and of the environment conditions.

Key words: foundation colony, ants, nest excavation.

\section{INTRODUÇÃO}

Anualmente, as colônias maduras de Atta produzem formigas aladas, machos e fêmeas, que abandonam a colônia na qual foram produzidas para formarem novas colônias e, dessa forma, continuarem

IMirmecoLab, Pós-graduação em Ciências Biológicas (PPGCB), Universidade Federal de Juiz de Fora (UFJF), Rua José Lourenço Kelmer, s/n, 36036-900, Juiz de Fora, MG, Brasil. E-mail: camargobotucatu@yahoo.com.br. *Autor para correspondência.

"Laboratório de Insetos Sociais-Praga, Departamento de Produção Vegetal, Faculdade de Ciências Agronômicas, Universidade Estadual Paulista (UNESP), São Paulo, SP, Brasil. 
a perpetuação da espécie (FORTI \& BOARETTO, 1997). As revoadas ocorrem sempre depois de fortes chuvas, que oferecem condições favoráveis para a sobrevivência inicial do fungo coletado antes do voo nupcial (AUTUORI, 1942). Além disso, a chuva facilita a escavação inicial do canal vertical por parte da rainha, além de ser um fator que permite o sincronismo do voo. Após o voo nupcial, a fêmea da saúva Atta sexdens escava um canal vertical de cerca de $15 \mathrm{~cm}$ e uma câmara, dentro da qual fica encerrada, cuidando da cultura de fungo e da prole (AUTUORI, 1942; RIBEIRO, 1995; CAMARGO et al., 2011; FRÖHLE \& ROCES, 2012). O processo de construção do novo ninho é o período mais crucial da vida de uma colônia de formiga e os mecanismos que otimizam a sobrevivência durante essa fase são de grande valor (WILSON, 1971).

A fundação de colônias de $\boldsymbol{A t t a}$ sempre envolve grande esforço da rainha, única responsável pelo cultivo do fungo e pelos cuidados consigo mesma e com sua prole. Nesse período, ocorre o consumo de grande parte de suas reservas (DELLA LUCIA et al., 1995). MINTZER (1990) afirmou que a fundação desses ninhos demanda o maior investimento já observado entre rainhas das várias espécies de formigas. Dessa forma, pode-se explicar o grande tamanho atingido por estas, devido ao acúmulo de reservas energéticas pré-voo nupcial, para que possam gastá-lo durante a formação de suas colônias (FUJUHARA et al., 2012; CAMARGO \& FORTI, 2013). De acordo com HEINZE \& TSUJI (1995), dentre os principais fatores que determinam o sucesso das estratégias reprodutivas, parecem estar a distribuição espacial e temporal dos habitats e da disponibilidade de locais de nidificação, o ambiente. Entretanto, são poucos estudos que se endereçam a estudar a influência ambiental no desenvolvimento de colônias de formigas (VIEIRA-NETO \& VASCONCELOS, 2010), bem como isso afeta o status fisiológico da rainha, durante a fundação e estabelecimento do ninho.

$\mathrm{O}$ presente estudo investigou como o ambiente influencia o desenvolvimento das 20 colônias recém-fundadas de Atta sexdens rubropilosa, bem como isso afeta o status fisiológico da rainha. Para tanto, foram comparadas colônias de campo coletadas cinco meses após a revoada e colônias mantidas em laboratório desde o voo nupcial.

\section{MATERIAL E MÉTODOS}

Colônias de laboratório de Atta sexdens rubropilosa: A coleta foi realizada durante a revoada que ocorreu no dia 28 de Outubro de 2011, pela captura das rainhas que estavam fundando suas colônias nas proximidades da Universidade Federal de Juiz de Fora, Juiz de Fora, MG. As rainhas recémcoletadas foram armazenadas em recipientes plásticos com $11 \mathrm{~cm}$ de diâmetro, $8 \mathrm{~cm}$ de altura, contendo $1 \mathrm{~cm}$ de gesso no fundo, para manter umidade. As rainhas foram transportadas até o Mirmecolab, UFJF, Juiz de Fora. Foram acondicionadas à temperatura de $24 \pm 2^{\circ} \mathrm{C}$, umidade relativa de $80 \%$ e um fotoperíodo de 12 horas claro. Após a emergência das primeiras operárias, as colônias foram alimentadas ad libitum com folhas de Acalypha wilkesiana, até completar 5 meses de idade, para comparação com as colônias de campo. Foram utilizadas 10 colônias.

Colônias de campo de Atta sexdens rubropilosa: A coleta foi realizada pela escavação dos ninhos de 5 meses de idade, marcadas com estacas no dia da revoada. Essa idade foi escolhida, pois muitos ninhos ainda não estão profundos, sendo de fácil escavação (CAMARGO \& FORTI, 2013). As escavações foram realizadas manualmente, utilizandose chibanca e espátulas, consistindo de uma abertura de trincheiras rasas de aproximadamente $1 \mathrm{~m}$ de largura por $1 \mathrm{~m}$ de profundidade, alargadas em direção ao lado da entrada do ninho até aparecimento da câmara. Todo o conteúdo foi recolhido e armazenado em recipientes plásticos com $11 \mathrm{~cm}$ de diâmetro, $8 \mathrm{~cm}$ de altura, contendo $1 \mathrm{~cm}$ de gesso no fundo, para manter a umidade. $\mathrm{O}$ material foi transportado até o Mirmecolab, UFJF, Juiz de Fora. Foram utilizadas 10 colônias.

Desenvovimento das colônias de campo e laboratório: o conteúdo foi levado ao laboratório e contabilizada a população de operárias pequena, média e grande (largura da cabeça 0,5 a 2,5mm, conforme CAMARGO \& FORTI, 2013) e sua mensuração corporal (largura da cabeça e tíbia). Posteriormente, pesou-se a biomassa (larva, pupa, jardim de fungo e operárias pequenas, médias e grandes). As rainhas foram separadas para a determinação de lipídeos, visando a comparar o seu esforço de escavação.

Determinação de lipídeos: Foi utilizada uma adaptação ao método de extração de COOK et al. (2010), em que os insetos foram imersos em solvente orgânico até atingirem um peso constante. Seguindo essa metodologia, as rainhas foram imersas em $10 \mathrm{ml}$ de pentano durante 24 horas, individualmente fechadas. Após esse período, o solvente foi removido e as rainhas permaneceram durante 24 horas a $70^{\circ} \mathrm{C}$ em uma estufa. Posteriormente, foi determinada sua massa em balança analítica. O procedimento foi repetido por 172 horas de extração. 
Análise estatística: O tamanho da população de colônias de campo e laboratório, peso populacional e do jardim de fungo foram comparados pelo Teste $t$ Student $(\alpha=0,05)$ e o teor de lipideos foram submetidos ao teste MannWhitney $(\alpha=0,05)$, utilizando o programa SigmaPlot 11.0. Para a largura da cabeça e comprimento da tíbia foi realizado uma correlação de Spearman, utilizando o programa R Development Core Team (2011).

\section{RESULTADOS}

As colônias de laboratório apresentaram em média uma maior população, quando comparadas às colônias de campo, diferindo estatisticamente para operárias pequenas $(\mathrm{t}=-6,87, \mathrm{~g} .1 .=18, \mathrm{P}<0,001)$, médias $(\mathrm{t}=-4,57$, g.l. $=18, \mathrm{P}<0,001)$, grandes $(\mathrm{t}=-$ 5,11, g.l. $=18, \mathrm{P}<0,001)$, pupas $(\mathrm{t}=-5,40$, g.l. $=18$, $\mathrm{P}<0,001)$ e larvas $(\mathrm{t}=-6,83$, g.l. $=18, \quad \mathrm{P}<0,001)$ (Figura 1). Consequentemente, o mesmo caso ocorreu para a biomassa, em que se detectaram diferenças significativas para o peso das rainhas $(\mathrm{t}=-3,64$, g.l. $=18, \mathrm{P}<0,001)$, operárias pequenas $(\mathrm{t}=$ $6,04$, g.l. $=18, \mathrm{P}<0,001)$, médias $(\mathrm{t}=-6,34$, g.l. $=18$, $\mathrm{P}<0,001)$, grandes $(\mathrm{t}=-6,17$, g.l. $=18, \mathrm{P}<0,001)$, pupas $(\mathrm{t}=-7,67, \mathrm{~g} .1 .=18, \mathrm{P}<0,001)$, e larvas $(\mathrm{t}=-8,6, \mathrm{~g} .1 .=18$, $\mathrm{P}<0,001$ ) (Figura 2).

O peso médio do jardim de fungo foi de

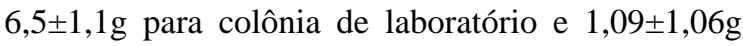
campo, diferindo estatisticamente entre eles $(\mathrm{t}=-$ 11,17, g.l.=18, $\mathrm{P}<0,001$ ) (Figura 3). O teor de lipídeos totais foi de $9,81 \pm 3,31 \%$ para rainhas de laboratório e $5,59 \pm 2,56 \%$ para as de campo, diferindo estatisticamente entre elas $(\mathrm{U}=12, \mathrm{P}<0,005)$ (Figura 4). Além disso, verificou-se uma correlação significativa entre os dados da medida da cabeça e da tíbia das operárias das colônias de campo e laboratório $(\mathrm{t}=167,720, \mathrm{rs}=0,98, \mathrm{P}<0,001)$.

\section{DISCUSSÃO}

Como esperado, as colônias de laboratório apresentaram um significativo desenvolvimento em relação as colônias de campo que sofreram a influência do ambiente. Primeiramente, a população de operárias de colônias de campo foi menor, sendo $48 \pm 19,3$ operárias pequenas, $16 \pm 0,4$ operárias médias e 9,7 $\pm 4,7$ operárias grandes (Figura 1). Esses valores são similares aos dados encontrados na literatura para colônias em seu habitat natural, por exemplo, CAMARGO \& FORTI (2013) verificaram que ninhos de 5 meses de Atta sexdens apresentavam cerca de

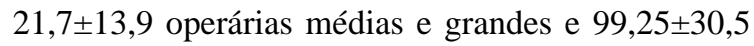
operárias pequenas. Por outro lado, PEREIRA-DASILVA (1975) verificou que o número de operárias em ninhos de 6 meses foi de 135,75 $\pm 54,26, \mathrm{~N}=4$, em colônias jovens de Atta sexdens rubropilosa. Cabe ressaltar, que em situação natural, muitas variáveis não são controladas, incluindo a quantidade e qualidade de alimento disponível ao forrageamento.

Dentro dessa perspectiva, VIEIRANETO \& VASCONCELOS (2010) ofereceram uma suplementação alimentar às colônias de Atta laevigata, as quais cresceram rapidamente e tiveram uma taxa de sobrevivência significativamente maior

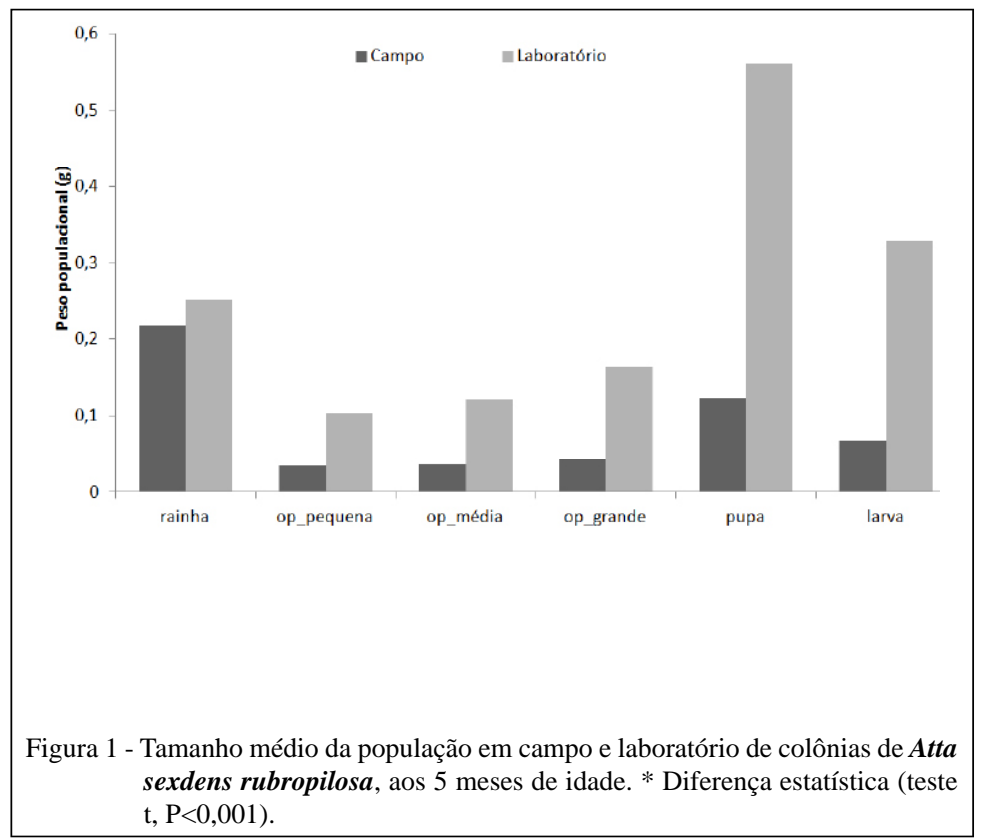

Ciência Rural, v.43, n.8, ago, 2013. 


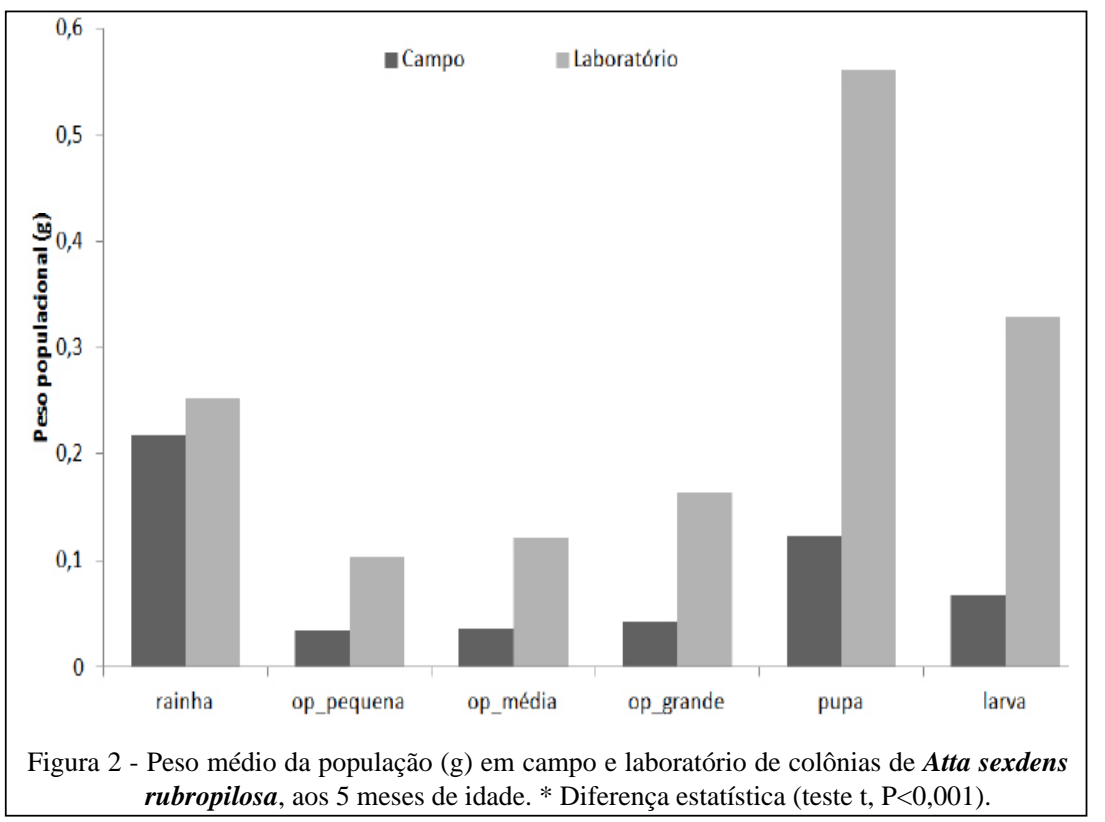

do que colônias controle. Os autores sugerem que o desempenho das formigas cortadeiras foi afetado pela disponibilidade de vegetais palatáveis em ambientes alterados. Em um estudo similar com a formiga cultivadora de fungo Trachymyrmex septentrionalis, SEAL \& TSCHINKEL (2008) verificaram que a suplementação de alimentos aumentou o crescimento do fungo simbionte, conduzindo a um aumento na quantidade de biomassa fúngica. Consequentemente, a quantidade de biomassa fúngica foi importante na determinação da quantidade da prole do que no tamanho da colônia. Nossos resultados corroboram o encontrado pelos autores, pois o peso do jardim de fungo foi superior em colônias de laboratório que receberam uma alimentação ad libitum, ao contrário das colônias de campo (Figura 3). Essa maior

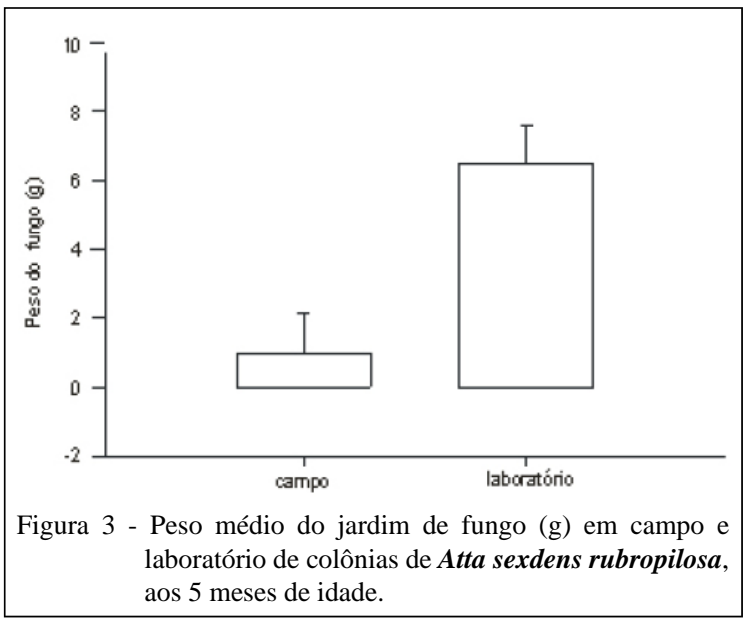

biomassa fungica foi responsável pelo aumento da população e de seu peso (Figuras 1 e 2).

A qualidade do alimento é extremamente importante, exemplificando, CAMARGO et al. (2008) ofertaram apenas um único vegetal por dois meses a colônias iniciais de Atta sexdens rubropilosa. Dentre as espécies vegetais, foi ofertado Acalypha spp., que promoveu o maior crescimento populacional, peso da rainha, postura, e taxa de produção de estáfilas (hifas intumescidas do jardim de fungo, fonte primária de alimento às larvas (BASS \& CHERRETT 1996). Mais uma explicação da grande diferença populacional entre as colônias de campo e laboratório de nosso estudo.

$\mathrm{O}$ peso médio das rainhas foi de $217,7 \pm 27,3 \mathrm{mg}$ para as de campo e $252,91 \pm 13,14 \mathrm{mg}$ para as de laboratório, valores abaixo do encontrado

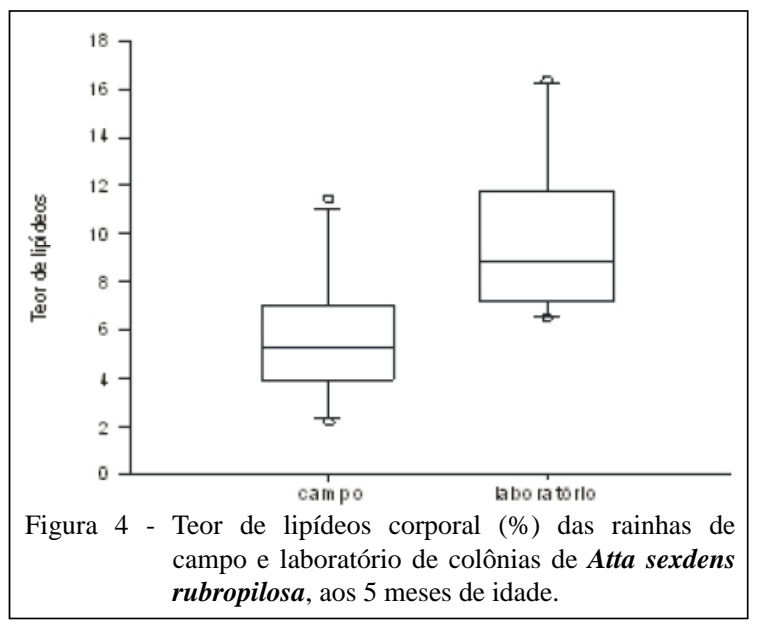


no dia da revoada, cerca de 700mg (CAMARGO et al., 2011; FUJIHARA et al., 2012). O teor de lipídeos foi de $9,81 \pm 3,31 \%$ para rainhas de laboratório e $5,59 \pm 2,56 \%$ para as de campo, bem abaixo das rainhas da revoada, de 33 a $40 \%$ para $\boldsymbol{A t t a}$ spp. (FUJIHARA et al., 2012; SEAL, 2009). Esse mesmo padrão de perda de massa corporal foi encontrado anteriormente por DELLA LUCIA et al. (1995), para colônias de laboratório de Atta sexdens e Atta laevigata. Essa perda de peso e de lipídeos totais demonstra que o acúmulo de reserva corporal por meio do armazenamento de lipídeos desempenhou um importante papel na história evolutiva da tribo Attini, evoluindo de uma fundação semiclautral para claustral (SEAL, 2009). De acordo com KELLER \& PASSERA (1989), as reservas de lipídeos são armazenados durante o período de maturação, ou seja, entre o tempo de emergência e de acasalamento, e serve como um combustível durante a época da fundação colônia para alimentar a rainha e sua prole.

As rainhas com fundação claustral não forrageiam e ficam enclausuradas em seu ninho, criando a sua prole pela metabolização de reservas corporais (PEETERS \& ITO, 2001; BROWN \& BONHOEFFER, 2003), como ocorre em Atta sexdens rubropilosa (AUTUORI, 1942). Possivelmente, a pressão de seleção atuante, para a evolução desse tipo de fundação, é o alto risco de mortalidade durante o forrageamento (HÖLLDOBLER \& WILSON, 1990), cuja, adaptação mais viável seria armazenar uma grande reserva energética em seu corpo.

Em relação ao tamanho das operárias, este foi similar em colônias de campo e laboratório, com 3 classes de tamanho. Esses resultados contrariam que a quantidade de alimento afeta a distribuição de tamanho das operárias (HÖLLDOBLER \& WILSON, 1990). As colônias de $\boldsymbol{A t t a}$ spp. apresentam uma plasticidade na distribuição da frequência de tamanho das operárias (WILSON, 1983). O autor realizou sociotomia em uma colônia adulta de $\boldsymbol{A t t a}$, de tal forma que a distribuição de tamanho das operárias fosse similar a uma colônia inicial. Surpreendentemente, o próximo ciclo de produção da prole foi igual a uma colônia inicial, independente da idade da colônia e da rainha. $\mathrm{O}$ autor conclui que o tamanho da colônia e, indiretamente, a quantidade de alimento são mais importantes que a idade.

Em conclusão, o estudo com Atta sexdens rubropilosa determinou que o ambiente exerce forte influência no desenvolvimento das colônias durante a fase de fundação, bem como sobre o status fisiológico das rainhas, sendo, portanto, a sobrevivência, desenvolvimento e sucesso da colônia reflexo das condições do ambiente e do esforço da rainha.

\section{CONCLUSÃO}

Conclui-se que o ambiente exerce forte influência no desenvolvimento das colônias durante a fase de fundação, bem como sobre o status fisiológico das rainhas.

\section{AGRADECIMENTOS}

R.S. Camargo agradece ao Conselho Nacional de Desenvolvimento Científico e Tecnológico (CNPq), pela bolsa concedida pela modalidade Pós doutorado Junior. L.C. Forti, ao $\mathrm{CNPq}$, pela bolsa produtividade à pesquisa (301917/2009-4). J.F.S. Lopes agradece à Fundação de Amparo à Pesquisa do estado de Minas Gerais (FAPEMIG) (APQ 00342-12). À Fundação de Amparo à Pesquisa do Estado de São Paulo (FAPESP), pelo apoio financeiro aos autores (Processos: 2007/04010-0 e 2007/07091-0) e ao CNPq (Processos 569962/2008-0 e 307335/2009-7).

\section{REFERÊNCIAS}

AUTUORI, M. Contribuição para o conhecimento da saúva (Atta spp - Hymenoptera- Formicidae). Arquivos do Instituto Biológico, v.13, p.137-150, 1942.

BASS, M.; CHERRETT J.M. Leaf-cutting ants (Formicidae, Attini) prune their fungus to increase and direct its productivity. Functional Ecology, v.10, p.55-61, 1996. Disponível em: <http:// europepmc.org/abstract/AGR/IND20560570/reload=0; jsessionid= HbUXayKdhXuvqp5o92Bi.12>. Acesso em: 06 dez. 2012. doi: $10.2307 / 2390262$.

BROWN, M.J.F.; BONHOEFFER, S. On the evolution of claustral colony founding in ants. Evolutionary Ecology Research, v.5, p.305-313, 2003. Disponível em: <http:// hdl.handle.net/2262/24942>. Acesso em: 06 dez. 2012 . doi: $10.2307 / 2390258$.

CAMARGO, R.S. et al. Growth of populations and fungus gardens of Atta sexdens rubropilosa (Hymenoptera, Formicidae) response to foraged substrates. Sociobiology, v.52, n.3, p.633643, 2003. Disponível em: <http://www.bv.fapesp.br/pt/producaocientifica/40398/growth-populations-fungus-garden-atta/>. Acesso em: 06 dez. 2012.

CAMARGO, R.S. et al. Digging effort in leaf-cutting ant queens (Atta sexdens rubropilosa) and its effects on survival and colony growth during the claustral phase. Insectes Sociaux, v.58, p.17-22, 2011. Disponível em: <http://dx. doi: 10.1007/s00040-010-01105>. Acesso em: 06 dez. 2012. doi: 10.1007/s00040-010-0110-5.

CAMARGO, R.S.; FORTI, L.C. Queen lipid content and nest growth in the leaf cutting ant (Atta sexdens rubropilosa). Journal of Natural History, v. 47, p. 65-73, 2013. Disponível em: <http://www.tandfonline. com/doi/abs/10.1080/00222933.2012.738836\#.Ua4li9I3u9E>. Acesso em 06 dez. 2012. doi:10.1080/00222933.2012.738836.

COOK, S.C.et al. Colony-level macronutrient regulation in ants: mechanisms, hoarding and associated costs. Animal Behaviour, v.79, p.429-437, 2010. 
DELlA LUCIA T.M.C. et al. Perda de peso de rainhas de Atta durante a fundação e o estabelecimento das colônias. Revista Brasileira Biologia, v.55, n.4, p.533-536, 1995.

FORTI, L.C.; BOARETTO, M.A.C. Formigas cortadeiras, biologia, ecologia, danos e controle. Botucatu-SP: UNESP, 1997. 70

FRÖHLE, K.; ROCES F. The determination of nest depth in founding queens of leaf-cutting ants (Atta vollenweideri): idiothetic and temporal control. Journal of Experimental Biology, v10 p.1642-1650. 2012.

FUJIHARA, R.T. et al. Lipids and energy contends in the bodies of queens of Atta sexdens rubropilosa Forel (Hymenoptera: Formicidae): pre and post nuptial flight. Revista Brasileira de Entomologia, v.56, n.1, p.7375, 2012 Disponível em: <http://www.scielo.br/scielo. php?script $=$ sci_arttext\&pid=S0085-56262012000100011 $>$. Acesso em: 06 dez. 2012. doi: <http://dx.doi.org/10.1590/ S0085-56262012005000015>.

HEINZE, J.; TSUJI K. Ant reproductive strategies. Researches on Population Ecolology, v.37, p.135-149, 1995. Disponível em: <http://link.springer.com/article/10.1007\%2FBF02515814? $\mathrm{LI}=$ true $>$. Acesso em: 04 mar. 2013. doi. 10.1007/BF02515814

HÖLlDOBLER, B.; WILSON, E.O. The ants. Cambridge: Harvard University, 1990. p.732.

KELLER, L.; PASSERA, L. Size and fat content of gynes in relation to mode of colony founding in ants (Hymenoptera: Formicidae). Oecologia, v.80, p.236-240, 1989. Disponível em: <http://link.springer.com/article/10.1007\%2FBF00380157>. Acesso em: 04 mar. 2013. 10.1007/BF00380157.

MINTZER, A. Foudress female weight and cooperative foudation in Atta leaf-cutting ants. In: VANDER MEER, R.K. et al. (Eds.), Applied myrmecology: a world perspective. Bouder: Westview, 1990. p.211-219.

PEETERS, C.; ITO, F. Colony dispersal and the evolution of queen morphology in social Hymenoptera. Annual Review Entomology, v.46, p.601-630, 2001. Disponível em: <http:// www.annualreviews.org/doi/abs/10.1146/annurev.ento.46.1.601>. Acesso em: 04 mar. 2013

PEREIRA-DA-SILVA, V. Contribuição ao estudo das Populações de Atta sexdens rubropilosa Forel, e Atta laevigata (Fr. Smith) no Estado de São Paulo. Studia Entomológica, v.18, n.1-4, p.2012-250, 1975.

R Development Core Team. R: A language and environment for statistical computing. Vienna: R Foundation for Statistical Computing, 2011. ISBN 3-900051-07-0, Disponível em <http:// www.R-project.org/>. Acesso em: 06 dez. 2012.

RIBEIRO, F.J.L. A escavação do solo pela fêmea da saúva (Atta sexdens rubropilosa). Psicologia-USP, v.6, n.1, p.75-93, 1995

SEAL, J.N.; TSCHINKEL, W.R. Food limitation in the fungusgardening ants, Trachymyrmex septentrionalis. Ecological entomology, v.33, n.5, p.597-607, 2008. Disponível em: <http:// onlinelibrary.wiley.com/doi/10.1111/j.1365-2311.2008.01010.x/ abstract>. Acesso em: 06 dez. 2012. doi: 10.1111/j.13652311.2008.01010.x

SEAL, J.N. Scaling of body weight and fat content in fungus gardening ant queens: does this explain why leaf-cutting ants found claustrally? Insectes Sociaux, v.56, p.135-141, 2009. Disponível em: <http://link.springer.com/article/10.1007\%2Fs00040-009-0002-8>. Acesso em: 06 dez. 2012. doi: 10.1007/s00040-009-0002-8.

VIEIRA, H.E.; VASCONCELOS, H.L. Developmental changes in factors limiting colony survival and growth of the leaf-cutters ants Atta laevigata. Ecography, v.33, p.538-544, 2010. Disponível em: 〈http://onlinelibrary. wiley.com/doi/10.1111/j.1600-0587.2009.05875.x/abstract〉. Acesso em: 06 dez. 2012. doi: 10.1111/j.1600-0587.2009.05875.x.

WILSON, E.O. The insect societies. Cambridge: Harvard University, 1971. 548p.

WILSON, E.O. Caste and division of labor in leaf-cutter ants (Hymenoptera, Formicidae: Atta). IV: Colony ontogeny of $\boldsymbol{A}$. cephalotes. Behavioral Ecology and Sociobiology, v.14, n.1, p.5560, 1983. Disponível em: <http://www.jstor.org/discover/10.2307 /459965 1 ?uid=3737664 $\&$ uid $=2 \&$ uid $=4 \&$ sid $=21101525404331>$. Acesso em: $06 \mathrm{dez} .2012$. 